\title{
The Twistor Connection and Gauge Invariance Principle
}

\author{
S. A. Merkulov \\ Department of Theoretical Physics, P. Lumumba Peoples' Friendship University, SU-117302 \\ Moscow, USSR
}

\begin{abstract}
It is shown that the twistor connection of the local twistor theory can be regarded as a gauge field whose Yang-Mills equations are equivalent to Bach equations of gravity.
\end{abstract}

\section{Introduction}

It is well known that the affine connection of Einstein's theory of gravity can be regarded as a gauge field. But consistent interpretation of the gravitation as a gauge field leads, strictly speaking, to non-Einstein theory, since the affine connection itself (not a metric tensor) turns out to be the basic field variable and the most natural gravitational Lagrangian should be quadratic in Riemann's curvature tensor [4]. Anyhow, two groups of field equations can be obtained in this approach:

(1) variations of the gravitational Lagrangian for the connection lead to (socalled) quasi-Maxwell equations for Riemann's curvature tensor (these are YangMills equations);

(2) variations of the gravitational Lagrangian for the metric tensor lead to (a generalization of) Einstein's equations (gravity field equations).

It should be stressed that in the case of the affine connection Euler-Lagrange equations of these variational principles (with any Lagrangian) are different, i.e. Yang-Mills equations do not coincide with gravity field equations.

In this work it is shown that the local twistor covariant derivative may be regarded as a gauge covariant derivative, resulting from the localization of the subgroup of the twistor group $\mathrm{SU}(2,2)$, which describes transformations of the local twistor components under conformal rescalings. In Sect. 2 the standard gauge transformation law of the Yang-Mills formalism is slightly modified to treat such cases, and in Sect. 4 it is proved that the twistor connection is a gauge field. In Sect. 5 a twistor connection Lagrangian is constructed and it is shown that the variational principle (1) (which gives sixty equations, fifty of which are zero identically) and the variational principle (2) (which gives ten equations) lead to the same conformally-invariant equations of gravity-vanishing of the Bach tensor.

The following index conventions are used: lower case Latin indices will be used for tensors, upper case Latin indices for spinors, and Greek indices for twistors. The sign convention for the curvature adopted here is that $\left[\nabla_{c}, \nabla_{d}\right] V_{b}=R_{\cdot b c d}^{a} V_{a}$, and $R_{a c b}^{c}=R_{a b}[2]$. 


\section{Gauge Transformations and Conformal Rescalings}

Consider some Lagrangian $L\left(g_{a b}, \psi, \nabla_{a} \psi\right)$, where $\psi$ is a section of some spinor bundle over a 4-dimensional pseudo-Riemannian manifold $\left(\mathbb{M}, g_{a b}\right)$, and $\nabla_{a}$ denotes the metric-compatible covariant derivative operator. Let the Lagrangian density be invariant under dilation of the metric (i.e. multiplication of the metric tensor at each point by a constant factor $k^{2}$ ) and action of some Lie group $G$ :

$$
L\left(k^{2} g_{a b}, G \psi, G \nabla_{a} \psi\right) k^{4} \sqrt{-g}=L\left(g_{a b}, \psi, \nabla_{a} \psi\right) \sqrt{-g} .
$$

To localize this symmetry ${ }^{1}$ :

$$
\begin{gathered}
g_{a b} \rightarrow \tilde{g}_{a b}=\Omega(x) g_{a b}, \\
\psi \rightarrow \tilde{\psi}=G(x) \psi,
\end{gathered}
$$

we may proceed as follows: for the invariance under independent actions of transformations (2.2) and (2.3) two compensating (gauge) fields should be introduced, but in the case of the invariance under simultaneous action of (2.2), (2.3), only one gauge field. It is the latter case we are interested in.

According to a general scheme of the Yang-Mills theory for the localization of this symmetry a gauge covariant operator is introduced [1]:

$$
\bar{\nabla}_{m} \psi \equiv \nabla_{m} \psi+\mathscr{A}_{m}(x) \psi
$$

where $\mathscr{A}_{m}(x)$ (called a gauge field or connection) is a set of functions on the manifold $\mathbb{M}$ with values in the Lie algebra of the group $G$.

The transformation of the covariant derivative operator $\nabla_{a}$ under conformal rescalings can be represented in the form:

$$
\nabla_{a} \psi \rightarrow \tilde{\nabla}_{a} \psi=\nabla_{a} \psi+\mathscr{B}_{a} \psi,
$$

where $\mathscr{B}_{a}(x)$ is a set of matrix-valued functions on the manifold $\mathbb{M}$. From now on possible spinor bundles and symmetry groups $G$ are restricted by the requirement that $\mathscr{B}_{a}$ belong to the Lie algebra of the group $G$ [the set of such bundles and groups is non-empty, for example, the local twistor bundle and the group $\mathrm{SU}(2,2)]$.

Proposition. If under transformations (2.2), (2.3) the gauge field $\mathscr{A}_{a}(x)$ transforms by the law:

$$
\mathscr{A}_{a} \rightarrow \tilde{\mathscr{A}}_{a}=G \mathscr{A}_{a} G^{-1}-\mathscr{B}_{a}-\left(\nabla_{a} G\right) G^{-1},
$$

and

$$
\widetilde{\nabla_{a}} G=\nabla_{a} G+\left[\mathscr{B}_{a}, G\right]
$$

the gauge covariant derivative (2.1) transforms as follows:

$$
\bar{\nabla}_{a} \psi \rightarrow \tilde{\bar{\nabla}}_{a}(G(x) \psi)=G(x) \bar{\nabla}_{a} \psi .
$$

The proof is straightforward.

1 Hereafter all quantities "living" on the pseudo-Riemannian manifold $\left(\mathbb{M}, \tilde{g}_{a b}\right)$ with the rescaled metric (2.2) will be equipped with a tilde over a kernel symbol 
Remark. Under gauge transformations (2.2), (2.3), and (2.6) the Lagrangian density $L\left(g_{a b}, \psi, \bar{\nabla}_{a} \psi\right) \sqrt{-g}[$ because of property (2.1)] is invariant:

$$
L\left(\tilde{g}_{a b}, G(x) \psi, \tilde{\bar{V}}_{a}(G(x) \psi)\right) \sqrt{-\tilde{g}}=L\left(g_{a b}, \psi, \bar{\nabla}_{a} \psi\right) \sqrt{-g} .
$$

Later we shall show that the twistor connection transforms by the law (2.6) and can be regarded as a gauge field in this sense.

\section{Local Twistors}

The formalism of local twistors was developed in $[2,3]$. Here a few of the important facts introduced in the just mentioned papers are merely summarized.

The local twistor space is by definition a complex vector bundle of range 4 over space-time with the structure group $\mathrm{SU}(2,2)$, whose typical fibre is the space of global (flat space-time) twistors. Each fibre may be thought of as the direct sum of a spin space and a conjugate dual spin space, but the exact way in which the local twistor splits to its spinor parts depends on the choice of the conformal scaling. Thus a local twistor $Z^{\alpha}$ at some point $Q$ can be represented with respect to the metric $g_{a b}$ by a pair of spinors $\left(\omega^{A}, \pi_{\dot{A}}\right)$ at $Q$. Under conformal rescaling $(2.2)$ the twistor $Z^{\alpha}$ changes its spinor representation according to the law:

$$
Z^{\alpha} \rightarrow \tilde{Z}^{\alpha} \equiv\left(\begin{array}{c}
\tilde{\omega}^{A} \\
\tilde{\pi}_{\dot{A}}
\end{array}\right)=\left(\begin{array}{cc}
\delta_{B}^{A}, & 0 \\
i \gamma_{\dot{A} B}, & \delta_{\dot{A}}^{\dot{B}}
\end{array}\right)\left(\begin{array}{c}
\omega^{B} \\
\pi_{\dot{B}}
\end{array}\right),
$$

where $\gamma_{\dot{A} B}=\Omega^{-1} \nabla_{\dot{A} B} \Omega$. For more explicit description of the relationship between twistors and spinors, projection and injection operators $e_{A}^{\alpha}, \mathrm{e}^{\alpha \dot{A}}, e_{\alpha \dot{A}}, e_{\alpha}^{A}$ have been introduced (analogs, in certain respects, of the Infeld-van der Waerdon symbols in the spinor formalism) in terms of which a local twistor can be written

$$
Z^{\alpha}=e_{A}^{\alpha} \omega^{A}+e^{\alpha \dot{A}} \pi_{\dot{A}}
$$

with the complex conjugate:

$$
\bar{Z}_{\alpha}=e_{\alpha \dot{A}} \bar{\omega}^{\dot{A}}+e_{\alpha}^{A} \pi_{A}
$$

The conformally invariant local twistor derivative has been defined by:

$$
\bar{\nabla}_{m} Z^{\alpha}=e_{\Lambda}^{\alpha}\left(\nabla_{M \dot{M}} \omega \omega^{A}+i \delta_{M}^{A} \pi_{\dot{M}}\right)+e^{\alpha \dot{A}}\left(\nabla_{M \dot{M}} \pi_{\dot{A}}-i P_{M B \dot{M} \dot{A}} \omega^{B}\right),
$$

where $^{2}$

$$
P_{M B \dot{M} \dot{B}} \equiv \frac{1}{2} R_{m b}-\frac{1}{12} R g_{m b}
$$

In conformally flat space-time the equation $\bar{\nabla}_{m} Z^{\alpha}=0$ has four linearly independent solutions over the field of complex numbers which correspond to global twistors [forming representation space for the pseudo-unitary group $\operatorname{SU}(2,2)$, this being locally isomorphic with the restricted conformal group of flat space-time].

2 We adopt the abstract index conventions [2] 


\section{The Local Twistor Connection as a Gauge Field}

For present purposes it is more convenient to rewrite the local twistor derivative in a matrix form:

$$
\bar{\nabla}_{m} Z^{\alpha}=\nabla_{M \dot{M}}\left(\begin{array}{c}
\omega^{A} \\
\pi_{\dot{A}}
\end{array}\right)+\left(\begin{array}{cc}
0 & i \delta_{M}^{A} \delta_{\dot{M}}^{\dot{B}} \\
-i P_{M \dot{M} B \dot{A}} & 0
\end{array}\right)\left(\begin{array}{l}
\omega^{B} \\
\pi_{\dot{B}}
\end{array}\right) .
$$

A matrix-valued field (completely defined by the metric structure)

$$
\mathscr{M}_{m \cdot \beta}^{\alpha}=\left(\begin{array}{cc}
0 & i \delta_{M}^{A} \delta_{\dot{M}}^{\dot{B}} \\
-i P_{M \dot{M} B \dot{A}} & 0
\end{array}\right)
$$

satisfies the following relations

$$
\mathscr{M}_{m \cdot \alpha}^{\alpha}=0, \quad \mathscr{M}_{m \cdot \beta}^{\alpha}=-\overline{\mathscr{M}_{m \cdot \alpha}^{\beta}},
$$

where the bar over $\mathscr{M}_{m \cdot \alpha}^{\beta}$ denotes the twistor conjugation. Therefore matrices $\mathscr{M}_{m}$ belong to su(2,2), the Lie algebra of SU(2,2), and (4.1) bears a formal resemblance to (2.4) if one puts $\psi=\left(\omega^{A}, \pi_{\dot{A}}\right)$ and $\mathscr{A}_{m}=\mathscr{M}_{m}$.

Proposition. Under transformations (2.2) and (2.3) with

$$
G=\left(\begin{array}{cc}
\delta_{B}^{A} & 0 \\
i \gamma_{\dot{A} B} & \delta_{\dot{A}}^{\dot{B}}
\end{array}\right) \quad \text { [see (3.1)], }
$$

the su(2,2)-valued field $\mathscr{M}_{m}(x)$ transforms as in (2.6) and $G$ automatically satisfies (2.7).

Proof. First of all it is necessary to find matrices $\mathscr{B}_{m}(x)$, defined by relations (2.5) which in the twistor space have the form [2]:

$$
\tilde{\nabla}_{m}\left(\begin{array}{c}
\omega^{A} \\
\pi_{\dot{A}}
\end{array}\right)=\nabla_{m}\left(\begin{array}{c}
\omega^{A} \\
\pi_{\dot{A}}
\end{array}\right)+\left(\begin{array}{cc}
\delta_{M}^{A} \gamma_{B \dot{M}} & 0 \\
0 & -\delta_{\dot{M} \gamma_{\dot{A} M}^{\dot{B}}}
\end{array}\right)\left(\begin{array}{c}
\omega^{B} \\
\pi_{\dot{B}}
\end{array}\right) .
$$

Thus the matrices $\mathscr{B}_{m}$ are

$$
\mathscr{B}_{m \cdot \beta}{ }^{\alpha}=\left(\begin{array}{cc}
\delta_{M}^{A} \gamma_{B \dot{M}} & 0 \\
0 & -\delta_{\dot{M}}^{\dot{B}} \gamma_{\dot{A} M}
\end{array}\right),
$$

and belong to $\mathrm{su}(2,2)$. Since under conformal rescalings [2]

$$
\widetilde{P}_{A B \dot{B} \dot{B}}=P_{A B \dot{A} \dot{B}}-\gamma_{\dot{A} B} \gamma_{\dot{B} A}+\nabla_{A \dot{A}} \gamma_{B \dot{B}},
$$

the proof is completed by a direct substitution of all necessary quantities into (2.6).

Therefore the twistor derivative (4.1) may be regarded as a gauge covariant derivative and the matrix-valued field $\mathscr{M}_{m}$ (called the twistor connection) as a gauge field, insuring invariance under action of the subgroup of $\mathrm{SU}(2,2)$, which describes the transformation of local twistor components under conformal rescalings.

It should be pointed out that the transformation of the analogously obtainable connection of another (torsion-free) conformally invariant twistor derivative (introduced in [3]) cannot be represented in the form (2.6), and, therefore, it cannot 
be regarded as a gauge field. In this respect the derivative (3.2) is distinguished among other possible definitions of the local twistor derivative (by the way, (3.2) is distinguished also in producing global twistors in the extreme case of conformally flat space-times [3]).

Since gauge transformations include rescalings of the metric, the standard form of the gauge field curvature

$$
\mathscr{F}_{m n}^{\prime} \equiv \nabla_{m} \mathscr{M}_{n}-\nabla_{n} \mathscr{M}_{m}+\left[\mathscr{M}_{m}, \mathscr{M}_{n}\right]
$$

is not gauge covariant in our case.

But it is worth noting that the standard Yang-Mills equations for the curvature (4.8) (also gauge non-invariant)

$$
\nabla^{m} \mathscr{F}_{m n}^{\prime}+\left[\mathscr{M}^{m}, \mathscr{F}_{m n}^{\prime}\right]=0
$$

lead (after a short calculation) to the following equations:

$$
\begin{gathered}
R_{a b}=0, \\
C_{a b[c d ; m]}=0,
\end{gathered}
$$

i.e. to the vacuum Einstein equations and vacuum Bianchi identities. Thus the gauge non-covariance of the standard Yang-Mills curvature (4.8) may be interpreted as a conformal non-invariance of the Einstein theory of gravity (in contradistinction to other zero mass fields).

As in the standard procedure of obtaining the gauge field curvature [1] the commutator of gauge covariant derivative operators acting on some twistor $Z=\left(\omega^{A}, \pi_{\dot{A}}\right)$ is formed:

$$
\left[\bar{\nabla}_{m}, \bar{\nabla}_{n}\right] Z=\left[\nabla_{m}, \nabla_{n}\right] Z+\left(\nabla_{m} \mathscr{M}_{n}-\nabla_{n} \mathscr{M}_{m}+\left[\mathscr{M}_{m}, \mathscr{M}_{n}\right]\right) Z,
$$

from which it immediately follows that the twistor connection curvature differs from the standard form (4.8) by the presence of the additional term $\mathscr{R}_{m n}$

$$
\mathscr{F}_{m n}=\nabla_{m} \mathscr{M}_{n}-\nabla_{n} \mathscr{M}_{m}+\left[\mathscr{M}_{m}, \mathscr{M}_{n}\right]+\mathscr{R}_{m n},
$$

arising from the non-commutativity of Riemann's covariant derivative operators.

Making use of the following relations [3]:

$$
\begin{aligned}
& {\left[\nabla_{M \dot{M}}, \nabla_{N \dot{N}}\right] \omega^{A}=\left(\varepsilon_{\dot{M} \dot{N}} \psi^{A}{ }_{B M N}-\delta_{M}^{A} P_{N B \dot{N} \dot{M}}+\delta_{N}^{A} P_{M B \dot{M} \dot{N}}\right) \omega^{B},} \\
& {\left[\nabla_{M \dot{M}}, \nabla_{N \dot{N}}\right] \pi_{\dot{A}}=\left(\varepsilon_{N M} \bar{\psi}^{\dot{B}} \dot{A} \dot{M} \dot{N}+\delta_{\dot{M}}^{\dot{B}} P_{N M \dot{N} \dot{A}}-\delta_{\dot{N}}^{\dot{B}} P_{M N \dot{M} \dot{A}}\right) \pi_{\dot{B}},}
\end{aligned}
$$

the su(2,2)-valued field $\mathscr{R}_{m n}$ turns out to be of the form:

$$
\mathscr{R}_{m n}=\left(\begin{array}{lll}
\varepsilon_{\dot{M} \dot{N}} \psi_{B M N}^{A}-\delta_{M}^{A} P_{N B \dot{N} \dot{M}}+\delta_{N}^{A} P_{M B \dot{M} \dot{N}}, & 0 \\
0, & \varepsilon_{N M} \bar{\psi}^{\dot{B}} \dot{A} \dot{M} \dot{N} & +\delta_{\dot{N}}^{\dot{B}} P_{N M \dot{N} \dot{A}}-\delta_{\dot{N}}^{\dot{B}} P_{M N \dot{M} \dot{A}}
\end{array}\right) .
$$

Substitution of (4.11) into (4.10) implies the following explicit form of the curvature:

$$
\mathscr{F}_{m n}=\left(\begin{array}{cc}
\varepsilon_{\dot{M} \dot{N}} \psi^{A}{ }_{B M N}, & 0 \\
-i\left[\varepsilon_{\dot{M} \dot{N}} \nabla_{\dot{A}}^{R} \psi_{R B M N}+\varepsilon_{M N} \nabla_{B}^{\dot{R}} \bar{\psi}_{\dot{R} \dot{M} \dot{N} \dot{A}}\right], & -\varepsilon_{M N} \bar{\psi}^{\dot{B}} \dot{A} \dot{M} \dot{N}
\end{array}\right) .
$$


The local twistor $C_{\lambda \varrho \beta}^{\mu \sigma \alpha}$, defined by

$$
C_{\lambda \varrho \beta}^{\mu \sigma \alpha}=e_{\lambda}^{M} e_{\varrho}^{N} e^{\mu \dot{M}} e^{\sigma \dot{N}} \mathscr{F}_{M \dot{M} N \dot{N} \beta}{ }^{\alpha},
$$

coincides with the curvature twistor, introduced in [2,3]. Straightforward calculations show that under gauge transformations (2.2) and (2.3) the twistor connection curvature transforms as

$$
\mathscr{\mathscr { F }}_{m n} \rightarrow \widetilde{\mathscr{F}}_{m n}=G \mathscr{\mathscr { F }}_{m n} G^{-1}
$$

\section{The Yang-Mills Equations for the Twistor Connection}

The Lagrangian of any gauge field must be a scalar, and the Lagrangian density, a gauge invariant.

The Killing metric, defined on $\operatorname{su}(2,2)$ by

$$
\langle A, B\rangle \equiv \frac{1}{2}\left(A^{\alpha}{ }_{\beta} \bar{B}_{\alpha}{ }^{\beta}+B^{\alpha}{ }_{\beta} \bar{A}_{\alpha}{ }^{\beta}\right)
$$

is a gauge invariant:

$$
\left\langle G A G^{-1}, G B G^{-1}\right\rangle=\langle A, B\rangle .
$$

Since under conformal rescalings

$$
\begin{gathered}
\sqrt{-g} \rightarrow \sqrt{-\tilde{g}}=\Omega^{4} \sqrt{-g}, \\
g^{m n} \rightarrow \tilde{g}^{m n}=\Omega^{-2} g^{m n},
\end{gathered}
$$

it is easily seen that the integrand of the functional

$$
S=\frac{1}{4} \int\left\langle\mathscr{F}_{m n}, \mathscr{F}_{p q}\right\rangle g^{m p} g^{n q} \sqrt{-g} d^{4} x
$$

is a scalar and a gauge invariant. It has the same form as in the standard YangMills formalism (in contradistinction to the form of the curvature and the gauge transformation law of the twistor connection).

There are two variational principles with the functional (5.1):

(1) We may vary (5.1) for the twistor connection $\mathscr{M}_{m}$, regarding it as being independent of the metric tensor, i.e. $S=S\left(\mathscr{M}_{m}\right)$;

(2) Taking into consideration the internal metric structure of the twistor connection (4.2), we may vary (5.1) for the metric tensor, i.e. $S=S\left(g_{m n}\right)$. Let us start with the first variational principle, since it is much more in the spirit of the YangMills theory. Though the twistor connection curvature differs from the usual one by the presence of the term $\mathscr{R}_{m n}$, the latter will vanish under variations:

$$
\delta \mathscr{F}_{m n}=\nabla_{m} \delta \mathscr{M}_{n}-\nabla_{n} \delta \mathscr{M}_{m}+\left[\delta \mathscr{M}_{m}, \mathscr{M}_{n}\right]+\left[\mathscr{M}_{m}, \delta \mathscr{M}_{n}\right] .
$$

Hence a straightforward repeating of usual arguments [1] leads to

Proposition. The Euler-Lagrange equations of the variational principle (1) are

$$
\nabla^{m} \mathscr{F}_{m n}+\left[\mathscr{M}^{m}, \mathscr{F}_{m n}\right]=0 .
$$

Substitution of (4.12) into (5.2) leads to

$$
\nabla^{m} \mathscr{F}_{m n}+\left[\mathscr{M}^{m}, \mathscr{F}_{m n}\right]=i\left(\begin{array}{cc}
0, & 0 \\
Q_{N B \dot{A} \dot{N}}+\bar{Q}_{\dot{A} \dot{N} N B}, & 0
\end{array}\right)
$$


where

$$
Q_{A B \dot{A} \dot{B}} \equiv \nabla_{\dot{A}}^{M} \nabla_{\dot{B}}^{N} \psi_{M N A B}-P^{M N}{ }_{\dot{A} \dot{B}} \psi_{M N A B}
$$

Therefore the gauge invariant Yang-Mills equations for the twistor connection reduce to

$$
B_{a b} \equiv Q_{A B \dot{B} \dot{B}}+\bar{Q}_{\dot{A} \dot{B} A B}=0 .
$$

Transformation of (5.4) from a spinor form to a tensor one results in the following expression for the tensor $B_{a b}$ :

$$
B_{a b}=\nabla^{c} \nabla^{d} C_{c a b d}-P^{c d} C_{c a b d} .
$$

The tensor $B_{a b}$, called the Bach tensor [5], is algebraically independent of the Weyl tensor:

$$
B_{a b}=\nabla^{m} \nabla_{(a} R_{b) m}-\frac{1}{3} \nabla_{a} \nabla_{b} R-\nabla^{m} \nabla_{m} P_{a b}+4 P_{a m} P_{b}^{m}-g_{a b} P_{m n} P^{m n}
$$

and, as it is readily seen, all vacuum solutions of Einstein's equations of gravity automatically satisfy Bach equations (5.4).

Under conformal rescalings (2.2) the Bach tensor transforms as a conformal density of weight $-2[3]: \widetilde{B}_{a b}=\Omega^{-2} B_{a b}$. From the spinor representation of the Bach tensor it is most easily seen that: $B_{a b}=B_{(a b)}, \nabla^{a} B_{a b}=0$.

For the realization of the second variational principle it is necessary to substitute into the functional (5.1) explicit expressions of the twistor connection curvature (4.12). In this case the Lagrangian will take the form:

$$
L=\frac{1}{4}\left\langle\mathscr{F}_{m n}, \mathscr{F}^{m n}\right\rangle=\frac{1}{8} C_{a b c d} C^{a b c d},
$$

where $C_{a b c d}$ is the Weyl tensor. This Lagrangian was used by Bach in his work [5], where he showed that

$$
\frac{2}{\sqrt{-g}} \frac{\delta(\sqrt{-g} L)}{\delta g^{a b}}=B_{a b} .
$$

Thus the extremal equations of both variational principles coincide (!) and lead to conformally invariant equations of gravity.

Acknowledgement. I would like to thank Professor N. V. Mitskiévič for helpful conversations.

\section{References}

1. Dubrovin, B.A., Novikov, S.P., Fomenko, A.T.: Modern geometry. Moscow: Nauka 1979 (in Russian)

2. Penrose, R., MacCallum, M.A.H.: Twistor theory: an approach to the quantisation of fields and space-time. Phys. Rep. 6 C, 241-315 (1972)

3. Dighton, K.: An introduction to the theory of local twistors. Int. J. Theor. Phys. 11, 31-43 (1974)

4. Konopleva, N.P., Popov, V.N.: Gauge fields. Moscow: Atomizdat 1980 (in Russian)

5. Bach, R.: Zur Weylschen Relativitätstheorie und der Weylschen Erweiterung des Krümmungstensorbegriffs. Math. Z. 9, 110 (1921)

Communicated by S. Hawking

Received July 21, 1983; in revised form November 28, 1983 
